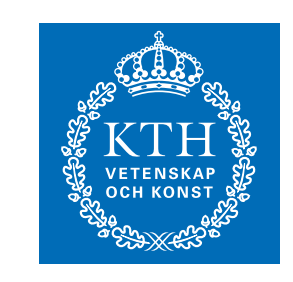

КTH Electrical Engineering

\title{
Bandwidth Efficient Compress-and-Forward Relaying Based on Joint Source-Channel Coding
}

(C)2011 IEEE. Personal use of this material is permitted. However, permission to reprint/republish this material for advertising or promotional purposes or for creating new collective works for resale or redistribution to servers or lists, or to reuse any copyrighted component of this work in other works must be obtained from the IEEE.

\section{RICARDO BLASCO-SERRANO, RAGNAR THOBABEN, AND MIKAEL SKOGLUND}

Stockholm 2011
Communication Theory Department
School of Electrical Engineering
KTH Royal Institute of Technology

IR-EE-KT 2011:002 


\title{
Bandwidth Efficient Compress-and-Forward Relaying Based on Joint Source-Channel Coding
}

\author{
Ricardo Blasco-Serrano, Ragnar Thobaben, and Mikael Skoglund \\ School of Electrical Engineering and ACCESS Linnaeus Centre \\ Royal Institute of Technology (KTH). SE-100 44 Stockholm, Sweden \\ Email: \{ricardo.blasco, ragnar.thobaben, mikael.skoglund\}@ee.kth.se
}

\begin{abstract}
We propose a new code design for compress-andforward relaying over bandlimited relay-to-destination channels. The main contribution of this paper is a code design based on joint (source-channel) coding and modulation that uses the correlation between the observations at the relay and the destination as protection against channel errors. This allows for relay nodes with reduced complexity, shifting most of the processing requirements to the destination node. Moreover, by using scalar quantizers with an entropy constraint our system provides remarkable performance in channel conditions where neither amplify-and-forward nor compress-and-forward efficiently exploit the presence of a relay node. Simulation results confirm the benefits of our proposed system.
\end{abstract}

\section{INTRODUCTION}

Cooperation is one of the present challenges in digital communications. Potential benefits are for example higher diversity or rate, helping to extend range or capacity of a communication network respectively. Several families of protocols for the classical cooperative model known as the relay channel exist. Two of the most significant ones are decode-and-forward (DF) and compress-and-forward (CF) [1]. Roughly speaking, in $\mathrm{DF}$ a relay node tries to decode the message from the source, re-encode (possibly a compressed version of) it and forward this information to the destination. DF is usually implemented using distributed channel coding schemes (e.g., turbo codes [2]). Clearly, the assumption of decodability at the relay restricts the scenarios where DF is useful. In contrast, in $\mathrm{CF}$ the relay listens to the message from the source and transmits a compressed version of its observation. In its simplest realization $\mathrm{CF}$ consists of transmitting a scaled version of the received signal. This is known as amplify-andforward (AF). Most of the work on practical implementations has focused on DF whereas only a few schemes for $\mathrm{CF}$ have been presented so far [3]-[7].

Besides the maximization of the performance (e.g., coverage extension, rate increase, etc.), relay designs should aim at minimizing the resources used by the relay nodes. In the related literature energy efficiency has received most of the attention. However, other equally important factors such as bandwidth (BW) efficiency have been widely neglected. Many relaying protocols have been proposed for the scenario in which both source and relay use the same resources simultaneously [3]. This leads to a high overall bandwidth efficiency but

This work was supported in part by the European Community's Seventh Framework Programme under grant agreement no 216076 FP7 (SENDORA) and VINNOVA. requires in turn accurate synchronization between transmitters and often full channel state information (CSI). However, this is not feasible unless the relay nodes have a high degree of sophistication. In this paper we propose a new relaying scheme with low complexity for the relay node and efficient usage of the $\mathrm{BW}$ on the relay-to-destination (RD) link. The relay avoids the use of complex distributed source coding tools by maintaining the correlation between its observation and that of the destination [8] to use it as redundancy for error protection. With the aid of its own observation the destination is able to recover the relay observation using iterative decoding. Finally, the source message is decoded using the two observations. As our results show, a significant performance gain can be achieved compared to previous implementations of $\mathrm{CF}$. Moreover, our system shows remarkable gains over DF and $\mathrm{AF}$ in the scenarios where neither of these protocols takes effectively advantage of the presence of the relay node.

This paper is organized as follows: in Section II we introduce the system model and discuss the issue of bandlimited relaying in the context of previously presented implementations of CF. In Section III we present our new scheme for $\mathrm{CF}$ relaying and design guidelines. Performance results and comparison to other alternatives are presented in Section IV. Section V concludes our work.

\section{BACKGROUND}

\section{A. Transmission model and notation}

In the scenario considered here (see Fig. 1) the source wants to convey a sequence of independent equally likely bits $\mathbf{U}$ to the destination. Communication is divided into two phases. In the first one the source performs channel coding $\left(\mathcal{C}_{s}\right.$ with rate $\left.R_{s}\right)$ on the information bits and modulation, and transmits the resulting symbols $\mathbf{X}_{S}$. Both relay and destination observe noisy versions of this transmitted signal, $\mathbf{Y}_{S R}$ and $\mathbf{Y}_{S D}$, respectively. In the second phase the relay processes its observation taking into account that the destination already has some side information about it available from the sourceto-destination (SD) link observation $\mathbf{Y}_{S D}$ [8] and transmits the resulting signal to the destination. Finally, using the noisy observation of the relay transmission $\mathbf{Y}_{R D}$ as well as $\mathbf{Y}_{S D}$, the destination tries to recover the information bits $\hat{\mathbf{U}}$.

This paper focuses on the scenarios where neither DF nor AF have a good performance due to low signal-to-noise ratios (SNR) on the source-to-relay (SR) and RD channels. All transmissions described here take place over additive white 


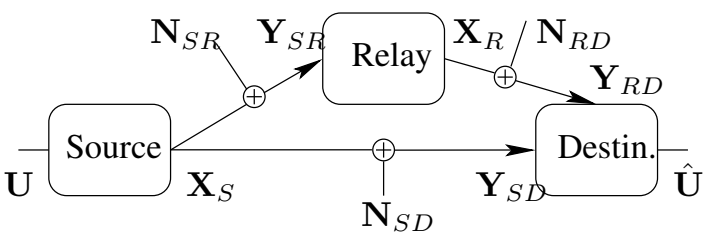

Fig. 1. Transmission model.

Gaussian noise (AWGN) channels. Moreover, the SD and RD channels are assumed to be orthogonal.

In this paper we assume that the number of channel uses in both communication phases is the same. It is therefore important to take into account the dimensionality of the modulations (e.g., real or complex baseband equivalents) used by the source and the relay. Even though the system to be presented here has no restriction on the dimensionalities of the modulations, all results here will be restricted to one-dimensional modulations on all links for simplicity. Moreover, extensions to allow for asymmetric bandwidth usages are straightforward.

Throughout this paper random variables are represented using upper case, e.g., $Z$, and their realizations using lower case, e.g., $z$. Scalars are represented with normal face $y$ and vectors with bold face $\mathbf{x}$. The element in position $i$ of a vector $\mathbf{x}$ is denoted by $x(i)$. A vector without its element in position $i$ is denoted by $\mathbf{x}(\underline{i})$.

\section{B. Coding for bandlimited channels}

In many practical scenarios the BW on the RD link may be severely limited. Common examples are multiple-relay networks where the resources to the destination are shared by all relays, simple relay architectures with narrowband amplifiers, etc. Relay designs for such scenarios need to consider this restriction yet providing substantial benefits over non-cooperative communications. It is well known that the performance of "ad hoc" solutions (e.g., combination of good channel codes and modulators) does not provide in general satisfactory results. The benefits of joint code and modulation design were already described by Ungerboeck [9]. With the advent of turbo and serially concatenated codes this idea was extended to take advantage of iterative decoding [10], [11].

\section{Related work}

Compression of the observation at the relay with low distortion requires high rates [8]. Moreover, coding for error protection on the RD channel increases the amount of bits to transmit per observed sample. Therefore, relaying requires either bandwidth expansion on the RD link or the use of BW efficient techniques.

Several practical implementations of CF have been proposed in the past. However, most of them use binary modulation during the second phase of the transmission [3], [4], [6], [7]. Hence their spectral efficiency is limited to one bit per channel use. In [5] relaying with superposition coding on the RD link was proposed. However, this system cannot achieve the performance of a Distributed Antenna System (DAS, optimal joint decoding of $\mathbf{Y}_{S D}$ and $\mathbf{Y}_{S R}$ ) regardless of the RD channel conditions (see [6]). Clearly, it is desirable to approach the performance of a DAS as the quality of the RD link increases.
While [6] takes into account this consideration it focuses on the BW expansion scenario. Moreover, the designs in [4]-[7] are not adaptable to the conditions on the RD channel unlike the system to be presented here.

\section{SYSTEM DESIGN}

It is a well known fact that the relay and the destination have correlated observations of the source message and that this correlation can be exploited by the relay to compress the observation reducing the number of bits per sample to be transmitted over the RD link for a given level of distortion [8]. Unfortunately, practical implementations with moderate complexity of this distributed source coding scheme have not appeared yet. Following the lines of [6] we propose to take into consideration not only the distributed source coding problem but also the effect of the RD channel. Instead of removing the correlation and then performing channel coding, with this new approach we take advantage of it to facilitate transmission to the destination. To realize this we describe here a new coding scheme based on joint source-channel coding and modulation at the relay and iterative decoding at the destination.

\section{A. Joint source-channel coding at the relay}

The relay node (see Fig. 2) consists of two modules (Source Code and Channel Code) concatenated through a bit interleaver ( $\pi$ in the figures). The source code consists of a Scalar Quantizer (SQ) ${ }^{1}$ followed by an outer code $\mathcal{C}_{o}$ with rate $R_{o}$. For practical reasons it is convenient to use SQs with $2^{m}$ quantization indices. In this way every index is represented using $m$ bits. In order to adapt the rate of transmission over the RD channel we propose to use SQs with a constraint on the entropy of the quantization indices. That is, the SQ has a fixed number of $2^{m}$ quantization indices and it is designed so that it has a bound on the entropy of its outputs $Y_{Q}{ }^{2}$. We refer to such a SQ as entropy constrained (ECSQ). That is, if $\mathscr{J}$ denotes the set of quantization indices then

$$
H\left(Y_{Q}\right)=-\sum_{j \in \mathscr{J}} p(j) \log _{2} p(j) \leq H_{0} \quad \text { bits/sample. }
$$

By varying $H_{0}$ it is possible to adjust the information rate and hence adapt the transmission to the RD channel conditions and make the joint source-channel code used by the relay decodable at the destination. It should be noted that constraining the entropy at the output of the quantizer increases the redundancy which can be exploited for error protection at the expense of a higher distortion between $\mathbf{Y}_{S R}$ and $\mathbf{Y}_{Q}$ [12]. The impact of the entropy constraint during iterative decoding will be analyzed in detail in Section III-D.

The channel code is formed by an inner code $\mathcal{C}_{i}$ with rate $R_{i}$ followed by a modulator with $M$ levels. In order to constrain the number of channel uses on the RD link to be the same as on the SR channel $M$ has to satisfy

$$
M \geq\left\lceil 2^{\frac{m}{R_{o} R_{i}}}\right\rceil .
$$

\footnotetext{
${ }^{1}$ The SQ operates on a sample-by-sample basis, where each sample has the same dimensionality as the modulation used by the source node.

${ }^{2}$ Throughout this text $Y_{Q}$ is used to refer to both quantization indices and reconstruction levels.
} 
In order to benefit from iterative decoding algorithms without sacrificing BW efficiency we use $R_{i}=1$ inner codes with $k=\log _{2}(M)$ input bits and one $M$-ary output symbol [10]. In this way we avoid symbol-to-bit transformations between the demapper and the decoder which are lossless in general. The design of the outer code and the inner code-mapper pair can be based on standard techniques such as EXIT charts [13].

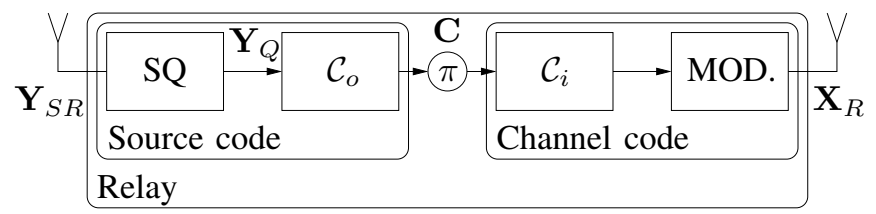

Fig. 2. Relay structure.

\section{B. Information combining at the destination}

Minimum bit error probability (BER) decoding of the source message at the destination amounts to obtaining $p\left(u \mid \mathbf{y}_{r d}, \mathbf{y}_{s d}\right)$. Taking into account the following Markov chain

$$
\mathbf{U} \rightarrow \mathbf{X}_{S} \rightarrow \mathbf{Y}_{S R} \rightarrow \mathbf{Y}_{Q} \rightarrow \mathbf{X}_{R} \rightarrow \mathbf{Y}_{R D},
$$

and the Data Processing Theorem [14] we have that

$$
I\left(U ; \mathbf{Y}_{Q} \mathbf{Y}_{S D}\right) \geq I\left(U ; \mathbf{Y}_{R D} \mathbf{Y}_{S D}\right),
$$

with equality if the RD transmission can be decoded without errors. Under this assumption then we have that

$$
p\left(u \mid \mathbf{y}_{r d}, \mathbf{y}_{s d}\right)=p\left(u \mid \mathbf{y}_{q}, \mathbf{y}_{s d}\right) .
$$

Equality in (1) suggests to decode the source message in two steps: first the relay transmission and then the source message. Optimal decoding as in (1) can be done using an a posteriori probability (APP) decoder that has $\mathbf{Y}_{Q}$ and $\mathbf{Y}_{S D}$ at its input. Using the conditional independence of $\mathbf{Y}_{Q}$ and $\mathbf{Y}_{S D}$ given $\mathbf{X}_{S}$ we have

$$
p\left(\mathbf{y}_{q}, \mathbf{y}_{s d} \mid \mathbf{x}_{s}\right)=p\left(\mathbf{y}_{q} \mid \mathbf{x}_{s}\right) p\left(\mathbf{y}_{s d} \mid \mathbf{x}_{s}\right),
$$

and since the SR and SD channels and the SQ are memoryless

$$
p\left(y_{q}, y_{s d} \mid x_{s}\right)=p\left(y_{q} \mid x_{s}\right) p\left(y_{s d} \mid x_{s}\right) .
$$

If we take logarithms this becomes

$$
L_{Y}^{C F}=L_{Y}^{Q}+L_{Y}^{S D} .
$$

and similarly for vectors.

\section{Iterative decoding}

Decoding of $\mathbf{U}$ based on APP processing of (2) requires perfect estimation of the quantization indices, i.e., $\hat{\mathbf{Y}}_{Q}=\mathbf{Y}_{Q}$. That is, transmission from the relay has to be decoded without errors. The optimal performance in terms of error probability (i.e., $\left.\operatorname{Pr}\left(\hat{Y}_{Q} \neq Y_{Q}\right)\right)$ is achieved by calculating $p\left(y_{q} \mid \mathbf{y}_{r d}, \mathbf{y}_{s d}\right)$. However, the presence of $\mathcal{C}_{S}$ at the source node and the structure of the relay node discourages this approach. Instead we propose iterative decoding of the component codes at the relay using a priori information on the information symbols (i.e., $\mathbf{Y}_{Q}$ ) obtained from the direct link observation $\mathbf{Y}_{S D}$. This is depicted in Fig. 3.

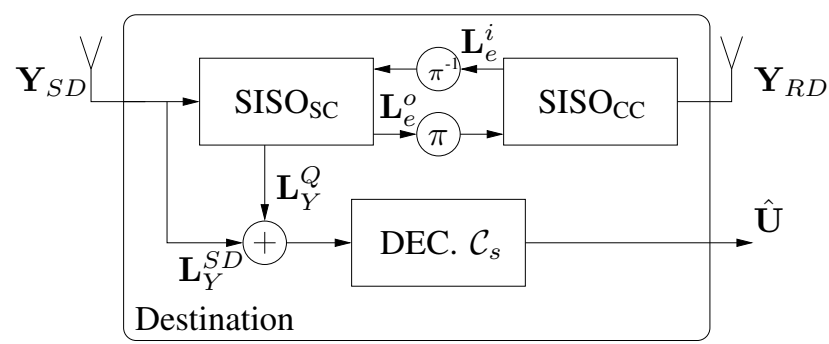

Fig. 3. Receiver structure at the destination.

In the first iteration the Soft-Input/Soft-Output (SISO) decoder for the channel code $\left(\mathrm{SISO}_{\mathrm{CC}}\right)$, which has no a priori information coming from the source code, obtains APP on the bits $\mathbf{C}$ [15]. The extrinsic parts of these probabilities are de-interleaved $\left(\pi^{-1}\right)$ and fed to the SISO decoder for the source code $\left(\mathrm{SISO}_{\mathrm{CS}}\right)$. This decoder uses this information in combination with the a priori information on $\mathbf{Y}_{Q}$ obtained from the SD link observation to obtain APPs on the symbols C. It passes the extrinsic part of these probabilities $\left(\mathbf{L}_{e}^{o}\right)$ to the $\mathrm{SISO}_{\mathrm{CC}}$ which uses them in combination with the RD link observations to generate new extrinsic information $\left(\mathbf{L}_{e}^{i}\right)$. Both decoders continue to iterate until convergence is achieved or a maximum number of iterations is reached [15]. We note here that decoding schedules that start with SISO $_{\mathrm{CS}}$ rather than $\mathrm{SISO}_{\mathrm{CC}}$ may result in earlier convergence. This, however, depends on the characteristics of the constituent codes.

\section{Direct link contribution and EXIT chart analysis}

As described above, proper processing of the direct-link observation is a key for decoding $\mathbf{X}_{R}$. Following the iterative decoding philosophy we propose to use $p\left(y_{q} \mid \mathbf{y}_{s d}\right)$ as a priori knowledge on the information symbols (i.e., $\mathbf{Y}_{Q}$ ) in the SISO $_{\mathrm{CS}}$. For time instant $n$ we have

$$
\begin{gathered}
p\left(y_{q}(n) \mid \mathbf{y}_{s d}\right)=\sum_{x_{s}(n)} p\left(y_{q}(n), x_{s}(n) \mid \mathbf{y}_{s d}\right) \\
=\sum_{x_{s}(n)} p\left(y_{q}(n) \mid x_{s}(n), \mathbf{y}_{s d}\right) p\left(x_{s}(n) \mid \mathbf{y}_{s d}\right) \\
=\sum_{x_{s}(n)} p\left(y_{q}(n) \mid x_{s}(n)\right) p\left(x_{s}(n) \mid \mathbf{y}_{s d}\right) .
\end{gathered}
$$

The last equality owes its validity to the conditional independence of $Y_{Q}(n)$ and $\mathbf{Y}_{S D}$ given $X_{S}(n)$. Equation (4) can be interpreted in terms of two contributions. The first term in the summation is determined by the SR channel statistics and the properties of the SQ. The second term is the APP on the $n^{t h}$ coded symbol given the direct-link observation and can be computed using a SISO algorithm.

If the structure of $\mathcal{C}_{S}$ is disregarded the following approximation is obtained

$$
\begin{aligned}
p\left(y_{q}(n) \mid \mathbf{y}_{s d}\right) & \approx p\left(y_{q}(n) \mid y_{s d}(n)\right) \\
& =\sum_{x_{s}(n)} p\left(y_{q}(n) \mid x_{s}(n)\right) p\left(y_{s d}(n) \mid x_{s}(n)\right),
\end{aligned}
$$

which is much simpler to compute. This might be of practical interest for codes $\mathcal{C}_{S}$ with a complex structure. The loss in 
performance incurred by the approximation corresponds to neglecting the information contained in $p\left(y_{q}(n) \mid \mathbf{y}_{s d}(\underline{n})\right)$ and can be predicted by examining the EXIT functions of the source code. An example of this degradation is shown in Fig. 4 for two different source codes. We can see that (5) yields less extrinsic information $I_{e}$ than (3) for the same level of a priori information $I_{a}$,

$$
I_{e}^{(3)}\left(I_{a}\right) \geq I_{e}^{(5)}\left(I_{a}\right)
$$

The benefits of considering the correlation between $\mathbf{Y}_{Q}$ (and hence $\mathbf{Y}_{R D}$ ) and $\mathbf{Y}_{S D}$ when decoding the same source code compared to a decoder that simply ignores it are easily observed from the EXIT functions in Fig. 4 (dash-dotted line vs. solid lines). Moreover, the differences in terms of convergence of the decoding algorithms when considering two ECSQs with different constraints $\left(H_{0}=1.5\right.$ and $\left.H_{0}=2\right)$ are also visible in the figure (solid vs. dashed lines). For both entropy constraints $\mathcal{C}_{O}$ is the same and only the SQ varies.

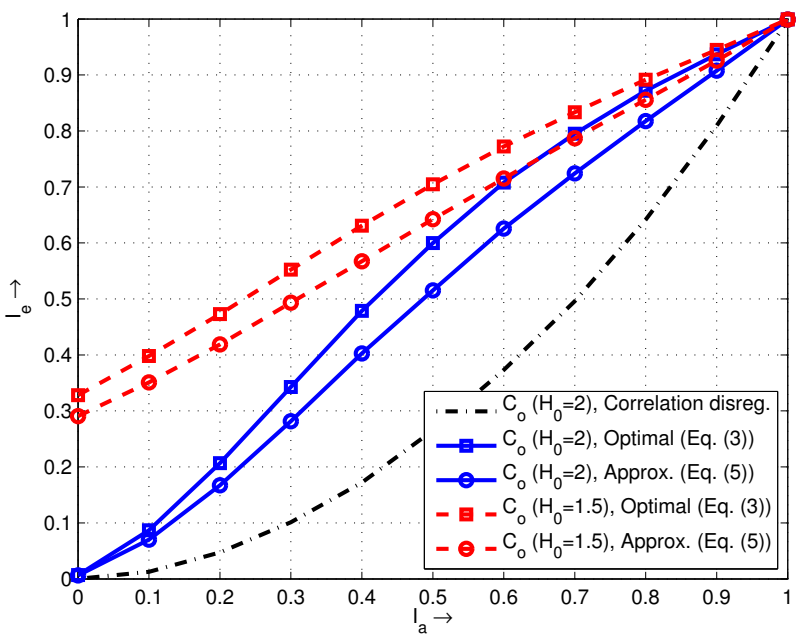

Fig. 4. EXIT functions for different realizations of the source code. The effect of the different degrees of exploitation of the correlation corresponds to the gap between curve pairs and to the case where the correlation is disregarded.

\section{Simulation RESULTS}

We have evaluated the performance of our proposed system with simulations. The source messages $\mathbf{U}$ consist of independent equally likely bits coded with $\mathcal{C}_{S}$ and BPSK modulated. The system presented here uses SQs with $m=1$ and $m=2$ and codes $\mathcal{C}_{o}$ with $R_{o}=1 / 2$ and $R_{o}=2 / 3$ respectively, and $\mathcal{C}_{i}$ with $R_{i}=1$. The parameters of the encoders and mappings used at the relay are summarized in Table I.

Fig. 5 shows the performance of the proposed $\mathrm{CF}$ in terms of end-to-end $\mathrm{BER}(\operatorname{Pr}(\hat{U} \neq U))$ when the $\mathrm{SNR}_{\mathrm{RD}}$ varies. In this example the convolutional encoder for $\mathcal{C}_{S}$ is $\left(1+D+D^{2}\right.$, $1+D^{2}$ ) and both SR and SD channels have the same quality. For comparison purposes we have also included the performance bound for the system in [5] as explained in [6] as well as the bounds for AF, non-cooperative and DAS schemes.

We describe first the performance of the proposed system with Lloyd-Max SQs [16] and iterative decoding based on (3). For $\mathrm{SNR}_{\mathrm{RD}}$ values between 1 and $9 \mathrm{~dB}$ the $\mathrm{CF}$ scheme uses a 1-bit SQ in combination with 4-PAM. For higher values of the

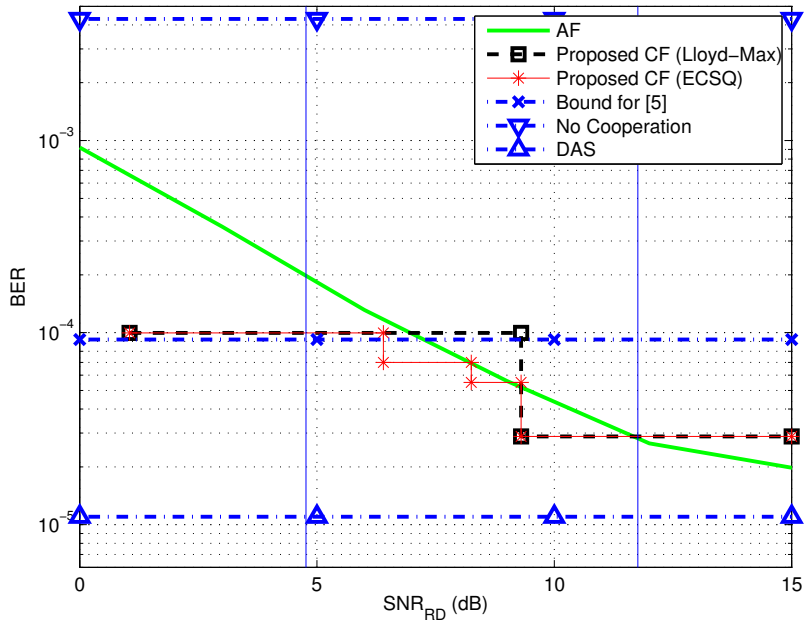

Fig. 5. BER for varying $\mathrm{RD}$ channel conditions $\left(\mathrm{SNR}_{\mathrm{SR}}=\mathrm{SNR}_{\mathrm{SD}}=3 \mathrm{~dB}\right)$

$\mathrm{SNR}_{\mathrm{RD}}$ a 2-bit SQs and 8-PAM are used. The step behavior at $\mathrm{SNR}_{\mathrm{RD}} \approx 9 \mathrm{~dB}$ is due to the convergence effect of the iterative decoder for the implementation with a 2-bit SQ. Fig. 5 shows that the combination of digital techniques (SQ and coding) can yield large gains in terms of $\mathrm{SNR}_{\mathrm{RD}}$ w.r.t. $\mathrm{AF}$. In this scenario the system with a 1-bit SQ outperforms AF in approximately $6 \mathrm{~dB}\left(\mathrm{BER}=10^{-4}\right)$. If a 2-bit $\mathrm{SQs}$ is used this gain is reduced to $2.5 \mathrm{~dB}\left(\mathrm{BER}=3 \cdot 10^{-5}\right)$. Note that the former has $C_{o}$ with $R_{o}=1 / 2$ whereas the latter has $R_{o}=2 / 3$.

In the range of $\mathrm{SNR}_{\mathrm{RD}}$ from 7 to $9 \mathrm{~dB}, \mathrm{AF}$ shows a better performance since the 1-bit SQ provides a too coarse observation of $\mathbf{Y}_{S R}$ and the 2-bit SQ scheme is still not decodable. However, as discussed in Sections III-A and III-D, it is possible to design systems in this range that converge and perform better than AF using 4-level ECSQs (see curve labeled as ECSQ). In this case the quantizers are designed using the algorithm described in [12] with all the other parameters the same as for the 2-bit scheme except for the mapping of the 8-PAM symbols (M1 vs. M2 in Table I). Using a SQ with $H_{0}=1.5$ (range $6-8 \mathrm{~dB}$ ) allows for decoding $4 \mathrm{~dB}$ earlier than the 2-bit Lloyd-Max SQ scheme an gives a SNR advantage around $2 \mathrm{~dB}$ over AF. In the case of $H_{0}=1.75$ (range $8-9 \mathrm{~dB}$ ) both gains are reduced to $1.25 \mathrm{~dB}$. This is due to the poor match between the EXIT functions of source and channel code. However, there exist other $\operatorname{codes} \mathcal{C}_{o}$ with the same complexity that result in better performance. In general, it is necessary to vary the code design. This example shows how to benefit from different degrees of CSI about the RD channel by using ECSQs.

We note here that using the minimum mean square error distortion criterion (Lloyd-Max or ECSQ) needs not yield optimal SQs in the way they are intended to be used here. If decoding of the relay message uses (5) instead of (3) losses in $\mathrm{SNR}_{\mathrm{RD}}$ of the order of $1-2 \mathrm{~dB}$ are observed.

Finally, in order to show the benefits of using the correlation between $\mathbf{Y}_{Q}$ and $\mathbf{Y}_{S D}$ as redundancy against errors on the RD channel we have included (as vertical lines) the minimum SNRs required for reliable transmission of 1 and 2 bits per channel use over an AWGN channel (i.e., 
TABLE I

SYSTEM PARAMETERS

\begin{tabular}{ccc}
\hline SQ & Outer Code & \multicolumn{1}{c}{ Inner Code } \\
\hline 1 bit & {$\left[\begin{array}{ccc}\frac{1}{1+D} & \frac{D}{1+D} \\
\frac{D}{1+D} & \frac{1}{1+D}\end{array}\right]$} \\
\hline 2 bits & {$\left[\begin{array}{ccc}1+D & 1 & D \\
D & 1 & 1+D\end{array}\right] \quad\left[\begin{array}{ccc}\frac{1}{1+D} & \frac{D}{1+D} & \frac{D}{1+D} \\
0 & 1 & \frac{1}{1+D} \\
0 & \frac{D}{1+D} & 1\end{array}\right]$} \\
\hline \hline
\end{tabular}

\begin{tabular}{c|cccccccc}
\hline \hline \multicolumn{10}{c}{ Mappings } \\
\hline Index: & 0 & 1 & 2 & 3 & 4 & 5 & 6 & 7 \\
\hline 4-PAM: & $3 A$ & $1 A$ & $-1 A$ & $-3 A$ & & & & \\
\hline 8-PAM M1: & $7 A$ & $5 A$ & $3 A$ & $1 A$ & $-1 A$ & $-3 A$ & $-5 A$ & $-7 A$ \\
8-PAM M2: & $7 A$ & $-3 A$ & $5 A$ & $-1 A$ & $3 A$ & $-7 A$ & $1 A$ & $-5 A$ \\
\hline \hline
\end{tabular}

$\left.C=\frac{1}{2} \log _{2}\left(1+\mathrm{SNR}_{\mathrm{RD}}\right)\right)$. The gap to modulation-constrained capacity (i.e., $M-\mathrm{PAM}$ ) is even larger.

Fig. 6 compares the end-to-end BER of the proposed coding scheme (1-bit Lloyd-Max SQ) against that of AF and of a conventional DF implementation with similar decoding complexity (Distributed Turbo Code (DTC) labeled as Stronger $P C C C$ in [2]). To allow for DF in the way described in [2], the encoder for $\mathcal{C}_{S}$ has been replaced by its systematic/recursive equivalent.

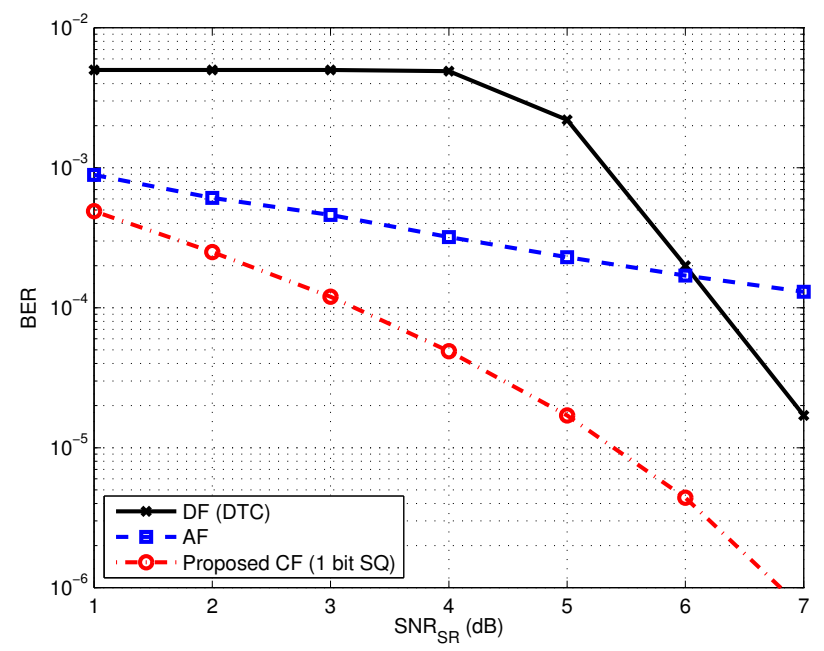

Fig. 6. Performance comparison for varying quality of the relay observation $\left(\mathrm{SNR}_{\mathrm{SD}}=\mathrm{SNR}_{\mathrm{RD}}=3 \mathrm{~dB}\right)$.

For low values of $\mathrm{SNR}_{\mathrm{SR}}$ both $\mathrm{CF}$ and $\mathrm{AF}$ show remarkable performance, for all three system are limited by the noise on the SR channel. However, as this noise is reduced the advantages of digital relaying over $\mathrm{AF}$ become visible. In fact as $\mathrm{SNR}_{\mathrm{SR}} \rightarrow \infty$ the performance of $\mathrm{AF}$ will tend to that of repetition coding. On the contrary, both $\mathrm{CF}$ and $\mathrm{DF}$ improve their performance with $\mathrm{SNR}_{\mathrm{SR}}$. However, DF requires a minimum SNR on the SR link in order to allow for DF cooperation resulting in a waterfall-like region. Stronger $\mathcal{C}_{S}$ codes yield a more distinct threshold, with DF mode never activated below it and always activated otherwise. On the contrary the proposed $\mathrm{CF}$ algorithm improves performance gradually for the pdf of $\mathbf{Y}_{S R}$ tends to concentrate around the transmitted symbols which leads to better quantization. We note here that more sophisticated DF schemes exist with steeper decreases of the BER, however, all of them suffer to some extent from the constraint of decodability on the SR link.
The same behavior is observed when increasing the $S N R_{R D}$. In this case the proposed $\mathrm{CF}$ can benefit from better RD link conditions and use a SQ with higher $H_{0}$ in the way it was described before.

\section{CONCLusion}

We have presented a new implementation of CF for cooperative communications with bandwidth constrained channels between relay an destination. Based on joint (source-channel) coding and modulation, the proposed design allows for cooperation with limited relay complexity. To achieve this we propose to keep the correlation between relay and destination observations and process it properly so it becomes useful as protection for errors introduced by the RD channel.

Our proposed design focuses on providing good performance in the cases where neither DF nor AF fully exploit the presence of the relay. In order to cover a wide range of SNR for the RD channel the system employs entropy-constrained SQs. In this way the relay can be designed to benefit from different degrees of CSI. Simulation results confirm that remarkable gains can be achieved with our system over previously existing cooperation protocols.

\section{REFERENCES}

[1] T. Cover and A. Gamal, "Capacity theorems for the relay channel," IEEE Trans. on Information Theory, vol. 25, no. 5, pp. 572-584, Sep. 1979.

[2] M. Valenti and B. Zhao, "Distributed turbo codes: towards the capacity of the relay channel," in IEEE 58th Vehicular Technology Conf., vol. 1, Oct. 2003, pp. 322-326.

[3] Z. Liu, V. Stankovic, and Z. Xiong, "Wyner-Ziv coding for the halfduplex relay channel," in IEEE International Conf. on Acoustics, Speech, and Signal Processing., vol. 5, Mar. 2005, pp. 1113-1116 Vol. 5.

[4] R. Hu and J. Li, "Practical compress-forward in user cooperation: Wyner-Ziv cooperation," in IEEE International Symposium on Information Theory, July 2006, pp. 489-493.

[5] T. Bui and J. Yuan, "A decode and forward cooperation scheme with soft relaying in wireless communication," in IEEE 8th Workshop on Signal Processing Advances in Wireless Communications., June 2007, pp. 1-5.

[6] R. Blasco-Serrano, R. Thobaben, and M. Skoglund, "Compress-andforward relaying based on symbol-wise joint source-channel coding," in IEEE International Conf. on Communications, June 2010.

[7] M. Uppal, Z. Liu, V. Stankovic, and Z. Xiong, "Compress-forward coding with BPSK modulation for the half-duplex gaussian relay channel," IEEE Trans. on Signal Proc., vol. 57, no. 11, Nov. 2009.

[8] A. Wyner and J. Ziv, "The rate-distortion function for source coding with side information at the decoder," IEEE Trans. on Information Theory, vol. 22, no. 1, pp. 1-10, Jan. 1976.

[9] G. Ungerboeck, "Channel coding with multilevel/phase signals," IEEE Trans. on Information Theory, vol. 28, no. 1, pp. 55 - 67, Jan. 1982.

[10] D. Divsalar, S. Dolinar, and F. Pollara, "Serial concatenated trellis coded modulation with rate-1 inner code," vol. 2, 2000, pp. $777-782$ vol.2.

[11] P. Robertson and T. Wörz, "Bandwidth-efficient turbo trellis-coded modulation using punctured component codes," IEEE Journal on Selected Areas in Communications, vol. 16, no. 2, pp. 206 -218, Feb. 1998.

[12] P. Chou, T. Lookabaugh, and R. Gray, "Entropy-constrained vector quantization," IEEE Trans. on Acoustics, Speech and Signal Processing, vol. 37, no. 1 , pp. $31-42$, Jan. 1989.

[13] A. Ashikhmin, G. Kramer, and S. ten Brink, "EXtrinsic Information Transfer functions: model and erasure channel properties," IEEE Trans. on Information Theory, vol. 50, no. 11, pp. 2657-2673, Nov. 2004.

[14] R. G. Gallager, Information Theory and Reliable Communication. New York, NY, USA: John Wiley \& Sons, 1968.

[15] J. Hagenauer, E. Offer, and L. Papke, "Iterative decoding of binary block and convolutional codes," IEEE Trans. on Information Theory, vol. 42, no. 2, pp. 429-445, Mar. 1996.

[16] S. Lloyd, "Least squares quantization in PCM," IEEE Trans. on Information Theory, vol. 28, no. 2, pp. 129-137, Mar. 1982. 